\title{
Lifetime and reliability improvements in modular multilevel converters using controlled circulating current
}

\author{
Nasiru B. Kadandani ${ }^{1}{ }^{10} \cdot$ Mohamed Dahidah $^{1} \cdot$ Salaheddine Ethni ${ }^{1} \cdot$ Musbahu Muhammad $^{2}$
}

Received: 16 April 2021 / Revised: 2 August 2021 / Accepted: 3 August 2021 / Published online: 25 August 2021

(c) The Author(s) 2021

\begin{abstract}
Circulating current has been an inherent feature of modular multilevel converters (MMC), which results in second-order harmonics on the arms currents. If not properly controlled, the circulating current can affect the lifetime and reliability of a converter by increasing the current loading, loss distribution, and junction temperature of its semiconductor devices. This paper proposes controlled circulating current injection as a means of improving the lifetime and reliability of an MMC. The proposed method involves modifying the reference modulating signals of the converter arms to include the controlled differential voltage as an offset. The junction temperature of the semiconductor devices obtained from an electro-thermal simulation is processed to deduce the lifetime and reliability of the converter. The obtained results are benchmarked against a case where the control method is not incorporated. The incorporation of the proposed control method results in a $68.25 \%$ increase in the expected lifetime of the converter and a 3.06\% increase on its reliability index. Experimental results of a scaled down laboratory prototype validate the effectiveness of the proposed control approach.
\end{abstract}

Keywords Modular multilevel converter (MMC) · Circulating current control (CCC) - Reliability $\cdot$ Lifetime $\cdot$ Rainflow algorithm

\section{Introduction}

Since its introduction in 2003 by Marquardt and Lesnicar [1], the modular multilevel converter (MMC) has been considered as a promising candidate for medium-to-high voltage conversion applications such as high-voltage direct current (HVDC) transmission systems [2-4], flexible alternating current transmission system (FACTS) [5], medium-voltage motor drives [6], wind energy conversion systems [7], and large-scale photovoltaic generation systems [8]. This wide range of applications is due in large part to some of the salient features associated with this converter such as its modular design and scalability to different power and voltage levels, low switching frequency, simple capacitor voltage balancing control, and simple realization of redundancy [9].

Nasiru B. Kadandani

n.b.kadandani2@newcastle.ac.uk

1 School of Engineering, Newcastle University, Newcastle upon Tyne, England, UK

2 School of Computing, Engineering and Digital Technologies, Teesside University, Middlesbrough, England, UK
One potential issue with the MMC is its circulating current, which needs to be properly controlled in order to improve the lifetime and reliability of the converter. Circulating current is a result of variations in the instantaneous voltages among the three phases that arise from voltage variations in the submodule (SM) capacitor voltages. Circulating current usually flows through the arms of a converter and distorts the arm currents by introducing second-order harmonics, adds extra losses to the converter, impacts its components ratings, and influences the amplitudes of the capacitor voltage ripples. The net effect of this is an increase in the thermal stress on the semiconductor switches, which results in reductions in the lifetime and reliability of the converter. Therefore, circulating current control (CCC) is one way to minimize thermal stress and improve the lifetime and reliability of an MMC.

Several methods have been proposed in the literature for CCC in MMCs for a variety of purposes [10-15]. However, none of these methods were focused on utilization for lifetime improvement. The authors of [14] proposed CCC as a means of improving the reliability of an MMC. However, the concept was only demonstrated on a Simulink model with only one SM per arm. In addition, it lacked 
submodule temperature regulation (SMTR), and the model was not experimentally validated. Furthermore, there was no attempt to estimate the impact of CCC on the lifetime of the converter.

This paper proposes a modified closed-loop CCC that was inspired by [15]. It is used as a means of improving the lifetime and reliability of a MMC. The modified control algorithm was based on injecting the differential voltage obtained from the closed-loop CCC as an offset to the reference modulating signals of the converter arms. The MMC control is based on the mathematical model presented in [16] along with an additional SMTR control loop as proposed in [17]. The purpose of the SMTR is to maintain equal thermal stress across all of the SMs to achieve equal reliability and lifetime expectancy. It also guarantees a more representative junction temperature profile for thermal analysis. The impact of CCC on the electrical behavior of an MMC was also studied.

In addition, this paper also investigates the impact of $\mathrm{CCC}$ on the lifetime and reliability of an MMC. Lifetime and reliability analyses in a power electronic converter (PEC) involve thermal stress control and lifetime estimation. The former was reported in $[18,19]$, while the latter was reported in $[20,21]$ using an accelerated lifetime test (ALT) and in $[22,23]$ using an analytical method. The ALT was based on subjecting the PEC switches to a more severe environmental condition by varying its junction temperature from its minimum value to its maximum value under a specified temperature swing $\Delta T_{j}$. The analytical method is based on analyzing the junction temperature profile of the switches using cycle counting methods such as the Hayes method, racetrack method, simple range counting, level counting, and peak counting [24].

In this paper, an analytical method based on a rainflow counting algorithm is used since it is computationally fast and can provide an average value [25]. Miners rule [26], which considers damage as a fraction of the life consumed by stress, is used to model the damage as a percentage of the consumed life based on loading history. This leads to the evaluation of the remaining useful lifetime (RUL) of the converter and its reliability index. The whole process is repeated for the case when CCC is incorporated. The obtained results show a great improvement in the lifetime and reliability index of the MMC. The effectiveness of the method is evaluated on a scaled down laboratory MMC prototype with four SMs per arm.

The remainder of this paper is organized as follows. Section 2 presents a schematic of an MMC, a control strategy using the proposed CCC injection, and the impact of the control method on MMC dynamics. Section 3 presents lifetime and reliability modeling and analysis. Section 4 reports simulation results and a discussion. In Sect. 5, experimental results are presented. Finally, some conclusions are drawn in Sect. 6.

\section{MMC control strategy}

\subsection{MMC circuit schematic}

Figure 1 shows a circuit schematic of a three-phase MMC. Each phase consists of two identical arms connected in series between the DC terminals. Each arm consists of $\mathrm{N}$-identical series-connected chopper cells called submodules (SM) and an arm inductor. An SM can be a full bridge or half bridge. In this paper, a half-bridge SM is used due to its simplicity in terms of component count. $V_{\mathrm{dc}}$ is the input DC voltage, $L_{\text {arm }}$ and $R_{\text {arm, }}$ are the arm inductance and its resistance, $i_{\text {up }}$ and $i_{\text {low }}$ are the currents through the upper and lower arms, $v_{\text {up }}$ and $v_{\text {low }}$ are their arm voltages, $i_{\text {circ }}$ is the circulating current, $L_{\text {load }}$ and $R_{\text {load }}$ represent an RL load, $i_{\mathrm{ac}}$ and $v_{\mathrm{ac}}$ are the output AC current and voltage, $i_{\mathrm{SM}}$ and $v_{\mathrm{SM}}$ are SM current and voltage, $C$ is the SM capacitor, and $v_{C}$ is the SM capacitor voltage. Table 1 shows the system parameters used in the simulation.

\subsection{Circulating current control in an MMC}

The circulating current $i_{\text {circ }}$ circulates through the arms of the converter without appearing on the output AC current. It is generated by the inner voltage differences in each phase of the converter in the form of a negative sequence with a frequency that is double that of the fundamental. It is made up of DC and AC components and can be expressed as:

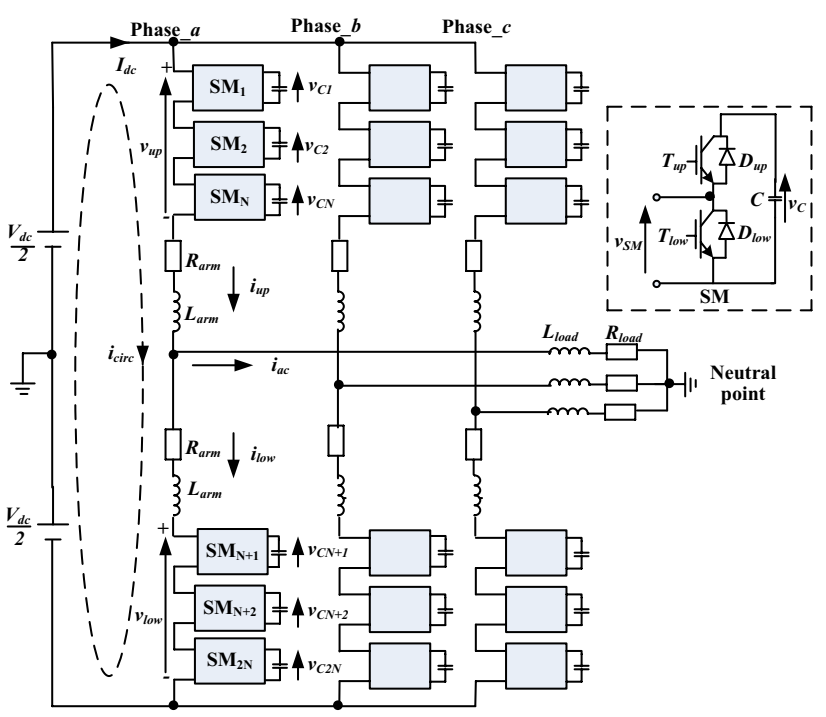

Fig. 1 Circuit schematic of a three-phase MMC 
Table 1 Simulation MMC system parameters

\begin{tabular}{ll}
\hline Parameter & Value \\
\hline Converter rating & $1 \mathrm{MVA}$ \\
Input DC voltage & $20 \mathrm{kV}$ \\
Output AC voltage reference & $10 \mathrm{kV}$ \\
Arm inductance & $10 \mathrm{mH}$ \\
Number of submodules per arm & 20 \\
Submodule capacitance & $10 \mathrm{mF}$ \\
Submodule capacitor voltage reference & $100 \mathrm{kV}$ \\
Carrier frequency & $2 \mathrm{kHz}$ \\
\hline
\end{tabular}

$i_{\mathrm{circ}}=i_{\mathrm{dc}}+i_{\mathrm{circ}_{\mathrm{ac}}}$.

The DC component $i_{\mathrm{dc}}$ is responsible for energizing the arms of the converter, and it maintains the SM capacitor voltages to their reference values. Meanwhile, the AC component $i_{\text {circ_ac }}$ is responsible for the capacitor voltage ripples. As such, the AC component of the circulating current has to be controlled to achieve an efficiency improvement, as well as loss and thermal stress minimization. The three-phase circulating currents are given as [15]:

$i_{\text {circ } \_\mathrm{a}}=\frac{I_{\mathrm{dc}}}{3}+i_{2 f} \sin \left(2 \omega_{o} t+\varphi_{o}\right)$,

$i_{\text {cir_ } \_ \text {b }}=\frac{I_{\mathrm{dc}}}{3}+i_{2 f} \sin \left(2 \omega_{o} t+\frac{2 \pi}{3}+\varphi_{o}\right)$,

$i_{\text {cir__c }}=\frac{I_{\mathrm{dc}}}{3}+i_{2 f} \sin \left(2 \omega_{o} t-\frac{2 \pi}{3}+\varphi_{o}\right)$,

where $I_{\mathrm{dc}}$ is the total DC current, $i_{2 f}$ is the peak value of the double-line frequency-circulating current, $\omega_{o}$ is the fundamental frequency, and $\varphi_{o}$ is the initial phase angle.

Equations (2)-(4) show that the AC parts of the circulating current contain a double-line frequency component that can be seen on the arm currents. This component adds undesirable losses, thermal stress, and current stress to the MMC, which can lead to reductions in the lifetime and reliability of the system.

In order to minimize these stresses, a modified CCC is proposed as means of improving the lifetime and reliability of a converter. The proposed controller eliminates the double-line frequency components in the arm current. It also minimizes the thermal stresses and current loading of the MMC semiconductor devices, which improves the lifetime and reliability of the converter.

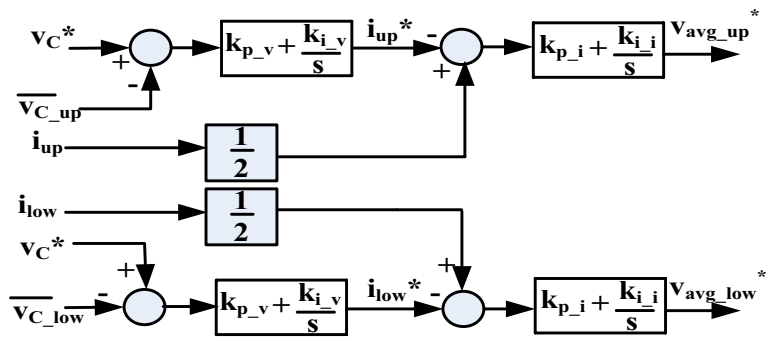

(a)

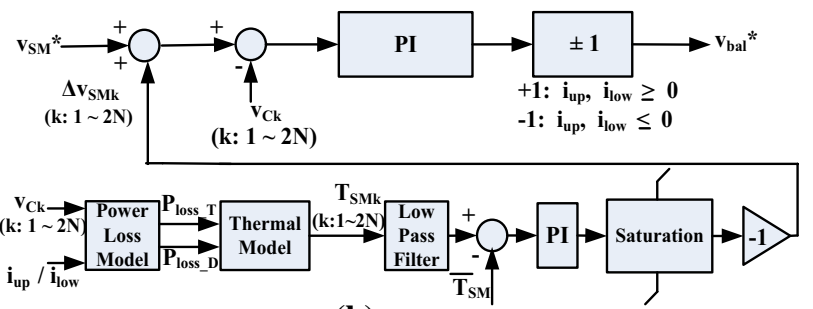

(b)

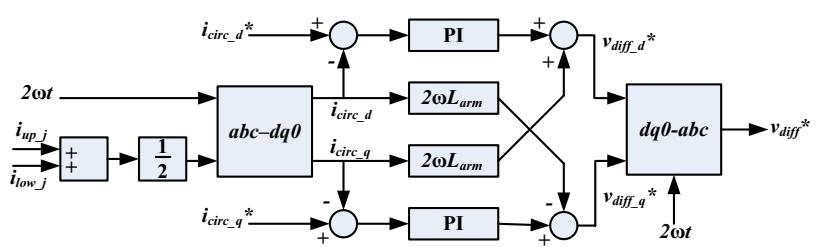

(c)

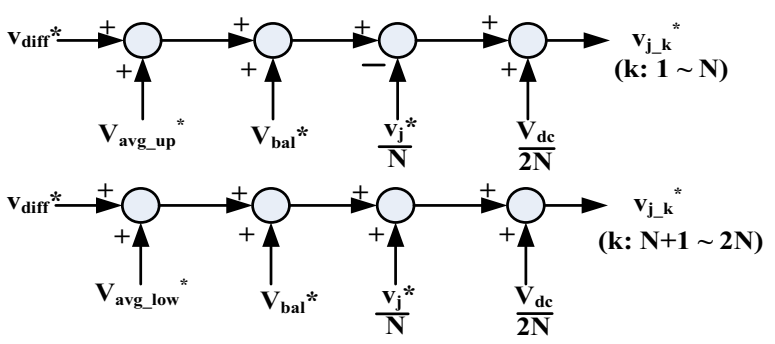

(d)

Fig. 2 Proposed control architecture of a MMC with controlled circulating current injection: a average DC voltage control; b SM voltage control and temperature regulation; $\mathbf{c}$ circulating current control; d modulation voltage synthesis

\subsection{Proposed control strategy}

In order to illustrate the modified control method, a system controller consisting of four parts is shown in Fig. 2. Figure 2a shows the average DC voltage control, which is meant to force the average DC capacitor voltage for each of the phases to follow its reference value. Figure $2 b$ shows individual DC voltage control integrated with SMTR. The aim of the individual DC voltage control is to force the DC capacitor voltages of individual SMs to follow their reference values. The purpose of the SMTR is to ensure equal 
reliability and lifetime expectancy of the SMs by equalizing their thermal stresses. Figure $2 \mathrm{c}$ is an $\mathrm{AC}$ circulating current controller, which is meant to suppress the double-line frequency components in the arm currents for each of the phases of the system and to minimize the current loading, losses, and temperatures of the semiconductor devices. Figure $2 \mathrm{~d}$ shows the modulation voltage synthesis.

\subsection{Impact of the proposed control strategy}

Table 2 gives a comparison of the advantages and disadvantages of a previous and the proposed CCC methods.
Simulated waveforms to verify the impact of the proposed $\mathrm{CCC}$ on the converter dynamics are shown in Fig. 3. The currents and multilevel voltages in the upper arm of the converter are shown in Fig. 3a, while their equivalents in the lower arm are shown in Fig. 3b. As can be seen, the CCC was able to transform the arm current waveforms into smooth sinusoidal shapes by cancelling the double-line frequency component that were distorting the waveforms. Meanwhile, the multilevel arm voltages were not affected. Figure $3 \mathrm{c}$ shows the three-phase output currents and voltages which seems to be unaffected by the control method. Figure $3 \mathrm{~d}$ shows the three-phase circulating current where

Table 2 Comparison of the proposed and previous control methods

\section{Previous method}

Based on centralized capacitor voltage balancing using a sorting algorithm

Fast sorting of the SM capacitor voltages in a converter with a large number of SMs is an issue

No dedicated thermal regulation loop

Not extended to the fatigue analysis/lifetime prediction of the converter
Proposed method

Based on a distributed capacitor voltage balancing algorithm using PI controllers

SM capacitor voltage balancing is achieved with some modifications to the modulating signal to provide good balancing

Combined with an SMTR for regulating the thermal stresses across all of the converter SMs to achieve equal reliability and lifetime expectancy

Extended to estimate the impact of the CCC on the lifetime and reliability of the converter

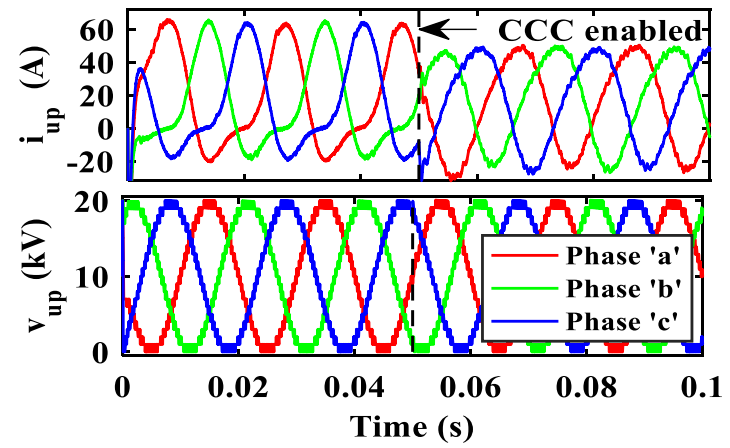

(a)

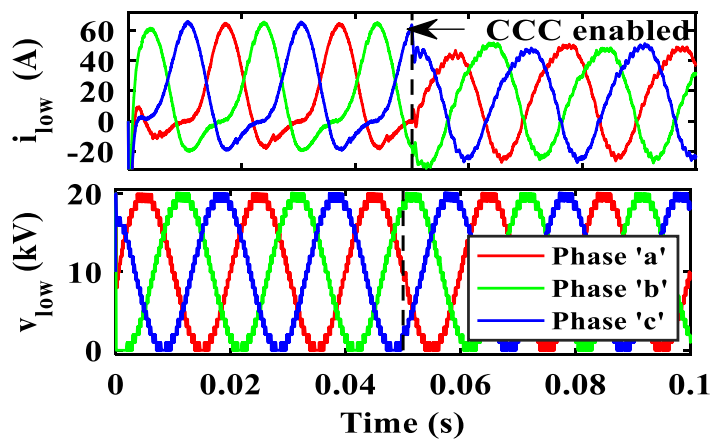

(b)
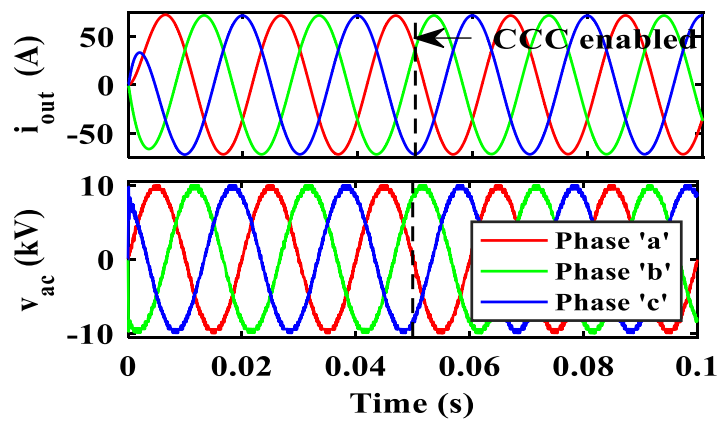

(c)

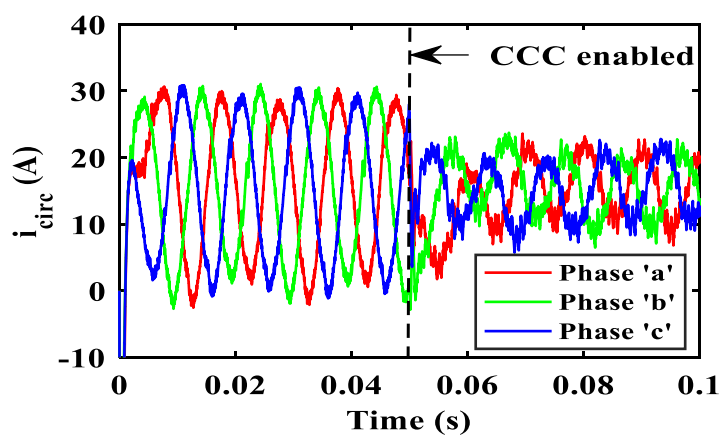

(d)

Fig. 3 Impact of the proposed control strategy on the electrical behavior of a converter: a currents and voltages in the upper arms; $\mathbf{b}$ currents and voltages in the lower arms; $\mathbf{c}$ output AC currents and voltages; $\mathbf{d}$ three-phase circulating current 
the effect of the controller reduces the peak-to-peak value of the current to less than half of its initial value.

\section{Lifetime and reliability modeling}

Power electronic converters (PECs) are vulnerable to failure especially when a system is operated in a harsh environment. The main critical stressors for PEC are temperature swings, voltage, humidity and vibrations [27]. The most affected components are semiconductor switches, capacitors, inductors, gate drives, printed circuit boards, resistors, fuses, and connectors. The stress coming from temperature variations has the highest impact on reliability [28], thus, providing adequate information for determining the failure rate of the system [29]. This is obvious considering the thermally induced stress arising from the differential thermal expansion of semiconductors. The resulting power cycles affect the lifetime and reliability of the PEC. In [30], a survey was conducted on over 200 products from 80 companies, and the results show that $31 \%$ of the respondents attributed the failures in PECs to semiconductor devices. According to [31], a total of 34\% of the failures in PECs comes from semiconductors and soldering followed by capacitors at $30 \%$.

\subsection{Temperature measurement and cycle counting}

Junction temperature is estimated based on an electro-thermal model consisting of power loss and thermal models. The semiconductor switch used in this paper is an FF75R12RT4 from Infineon [32]. The outputs of the thermal model are the junction temperatures of the semiconductor devices. After the first iteration, the calculated junction temperature values are updated into the power loss model for the next iteration. The fatigue analysis of devices with irregular loading profiles requires a proper algorithm for cycle counting. A common example is the rainflow algorithm as demonstrated in [33]. Once all of the temperature cycles are identified, the lifetime of the converter can be estimated by a conventional cycle-based lifetime model. The rainflow algorithm provides three parameters: the cycle amplitudes $T_{\mathrm{ja}}$, the ranges or mean value of the cycles $T_{\mathrm{jm}}$, and the number of the identified cycles $n$ that are stored in a matrix.

\subsection{Fatigue modeling}

The authors of [20] proposed a relationship between the lifetime (in number of cycles), the mean temperature, and the amplitude of the temperature. The expression, often referred to as the Coffin-Manson-Arrhenius model is given by:
$N_{\mathrm{f}}\left(T_{\mathrm{jm}}, \Delta T_{\mathrm{jm}}\right)=A * \Delta T_{\mathrm{j}}^{\alpha} * \exp \left(\frac{E_{\mathrm{a}}}{k_{\mathrm{B}} * T_{\mathrm{jm}}}\right)$,

where $N_{\mathrm{f}}$ is the expected number of cycles before failure, $T_{\mathrm{jm}}$ is the mean value of the temperature, $\Delta T_{\mathrm{jm}}$ is the amplitude (temperature range), $E_{\mathrm{a}}=9.89 * 10^{-20}(\mathrm{~J})$ is the activation energy, $k_{\mathrm{B}}=1.38066 * 10^{-23}(\mathrm{~J} / \mathrm{K})$ is the Boltzmann constant, and $\alpha$ and $A$ are constants that are module dependent.

The model is for $\Delta T_{\mathrm{jm}}$ values between 30 and $80 \mathrm{~K}$. However, they can be extrapolated to lower values.

\subsection{Damage modeling}

It is worth noting that (5) considers the magnitude of only one thermal cycle which is insufficient for lifetime estimation in real mission profiles such as the junction temperature of semiconductors. The extent to which the temperature swing is stressing the semiconductors for different magnitudes of thermal cycles needs to be evaluated. This can be achieved using the Palmgren-Miner rule, which assumes that damage accumulates linearly [34]. This law considers damage as a fraction of the life used by an event or a series of events [35]. The hypothesis behind Palmgren-Miner's rule is that the total damage is the sum of the particular damages caused by all of the identified cycles [36]. The damage caused by each distinguished thermal cycle contributes toward consuming the life of the system. The sum of the particular damages caused by each of the distinguished cycles represents the total damage, $D$.

$D=\sum_{i=1}^{n} D_{i}$

where $n$ is the number of identified temperature cycles determined from the loading history, as computed by the rainflow algorithm.

The damage due to an individual cycle $D_{i}$ is given by:

$D_{i}=\frac{n_{i}}{N_{f i}}$

where $N_{f i}$ is the number of cycle to failure.

The total damage is given by:

$D=\sum_{i=1}^{n} \frac{n_{i}\left(T_{\mathrm{jm}}, \Delta T_{\mathrm{jm}}\right)}{N_{f i}\left(T_{\mathrm{jm}}, \Delta T_{\mathrm{jm}}\right)}$.

Failure is expected when $D=1$, and no failure is expected when $D<1$. 


\subsection{Lifetime estimation}

After the damage is quantified based on the distinguished cycles, the RUL of the semiconductor $\mu$ can be calculated as the inverse of the damage multiplied by the time period during which the damage occurs:

$\mu=\frac{T_{o}}{D}$,

where $T_{o}$ is the interval of the time history.

\subsection{Reliability modeling}

The reliability of an item $R(t)$ is defined as the probability that the item will perform a required function without failure under stated conditions for a stated period of time [37].

$R(t)=e^{-\lambda t}$,

where $\lambda$ is the failure rate and $t$ is the mission time.

In this study, the RUL of the semiconductor device $\mu$ computed using (9) is assumed to be the mean time to failure (MTTF) of the semiconductor device of an MMC. Based on this assumption, the following can be written:

$\mathrm{MTTF}_{\mathrm{IGBT}}=\mu_{\mathrm{IGBT}}$.

Accordingly, the failure of the IGBT can be expressed in terms of its MTTF as:

$\lambda_{\mathrm{IGBT}}=\frac{1}{\mathrm{MTTF}_{\mathrm{IGBT}}}$.

According to (12), the reliability index of the IGBT $R_{\mathrm{IGBT}}$ can be computed as:

$R_{\mathrm{IGBT}}=e^{-\lambda_{\mathrm{IGBT}}}$.

The SM chopper cells in an MMC are serially connected. As such, the reliability of the MMC $R_{\mathrm{MMC}}$ can be modeled using series association reliability modeling based on the following expression [38]:

$R_{\mathrm{MMC}}=\prod_{i=1}^{N} R_{\mathrm{IGBT}}$.

However, it is worth noting that in this research, the SMTR loop has been incorporated into the individual DC capacitor balancing with the aim of equalizing the thermal stress among all the SMs. This is assumed to translate into equal reliability and lifetime expectancy of the SMs. Based on the foregoing, (14) can be rewritten as:

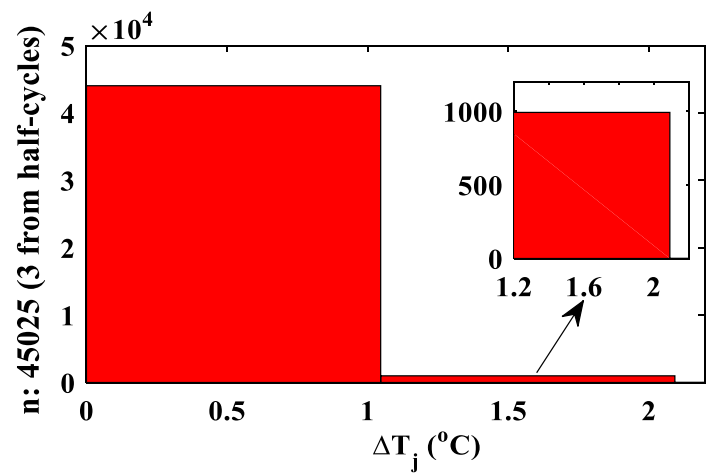

Fig. 4 Rainflow histogram of temperature swings among identified cycles without the proposed CCC

Table 3 Life Consumption of one switch of a converter without CCC

\begin{tabular}{lll}
\hline Parameter & Value & \\
\hline Temperature swing, $\Delta T_{\mathrm{j}}\left({ }^{\circ} \mathrm{C}\right)$ & $0 \leq \Delta T_{\mathrm{j}} \leq 1.04$ & $1.1<\Delta T_{\mathrm{j}} \leq 2.1$ \\
Mean temperature, $T_{\mathrm{jm}}\left({ }^{\circ} \mathrm{C}\right)$ & 59 & 57 \\
Number of identified cycles, $n$ & 44031.5 & 993.5 \\
Number of cycles to failure, $N_{\mathrm{f}}$ & $6.4026 \times 10^{15}$ & $2.0467 \times 10^{14}$ \\
Consumed life (Damage), $D_{i}(\%)$ & $6.8771 \times 10^{-10}$ & $4.8541 \times 10^{-10}$ \\
\hline
\end{tabular}

$R_{\mathrm{MMC}}=R_{\mathrm{IGBT}}^{N}$.

According to [39], the reliability of an MMC can be equated to the reliability of one arm due to symmetry. As such, the parameter $N$ in (15) is the number of SMs in one arm of the converter.

The failure rate of the MMC system can be calculated as:

$\lambda_{\mathrm{MMC}}=-\log \left(R_{\mathrm{MMC}}\right)$.

The MTTF of the MMC MTTF $_{M M C}$ can be obtained as:

$\mathrm{MTTF}_{\mathrm{MMC}}=\frac{1}{\lambda_{\mathrm{MMC}}}$.

\section{Results and discussion}

\subsection{Case I: results and analysis without CCC}

Figure 4 shows a rainflow histogram of the temperature swing among the identified cycles. Results for the life consumption of the semiconductor switch under this condition are given in Table 3 . The total life consumption of one IGBT of the converter is the sum of the accumulated damages due to all of the temperature swings. The accumulated damage is evaluated as $1.1731 \times 10^{-9} \%$, which yields an expected lifetime of 270.3077 years. By letting the RUL be the MTTF 
Table 4 Lifetime and reliability of a converter without CCC

\begin{tabular}{llr}
\hline Parameter & Designation & \multicolumn{1}{c}{ Value } \\
\hline MTTF for IGBT & $\mu_{\text {IGBT }}$ (years) & 270.3077 \\
IGBT failure rate & $\lambda_{\text {IGBT }}$ & 0.0037 \\
IGBT reliability & $R_{\text {IGBT }}$ & 0.9963 \\
MMC reliability & $R_{\text {MMC }}$ & 0.9285 \\
MMC failure rate & $\lambda_{\text {MMC }}$ & 0.0742 \\
MTTF for MMC & $\mu_{\mathrm{MMC}}$ (years) & 13.4771 \\
\hline
\end{tabular}

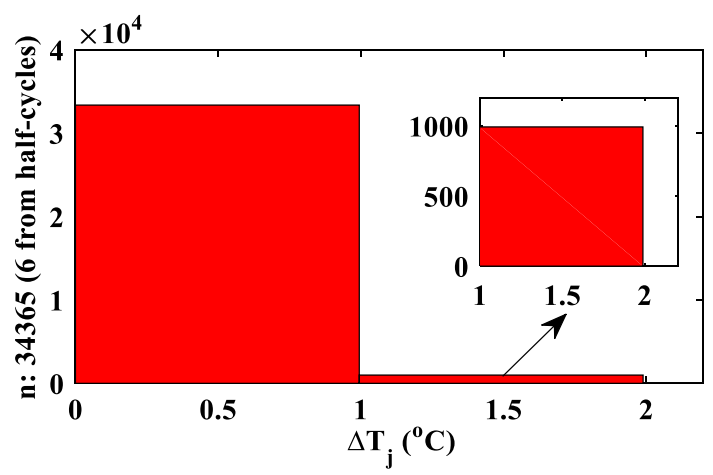

Fig. 5 Rainflow histogram of temperature swings among identified cycles with the proposed CCC

Table 5 Life consumption of one switch of a converter with CCC

\begin{tabular}{lll}
\hline Parameter & Value & \\
\hline Temperature swing, $\Delta T_{\mathrm{j}}\left({ }^{\circ} \mathrm{C}\right)$ & $0 \leq \Delta T_{\mathrm{j}} \leq 1$ & $1<\Delta T_{\mathrm{j}} \leq 1.99$ \\
Mean temperature, $T_{\mathrm{jm}}\left({ }^{\circ} \mathrm{C}\right)$ & 57 & 56 \\
Number of identified cycles, $n$ & 33371.5 & 993.5 \\
Number of cycles to failure, $N_{\mathrm{f}}$ & $8.8917 \times 10^{15}$ & $2.9629 \times 10^{14}$ \\
Consumed life (Damage), $D_{i}(\%)$ & $3.7531 \times 10^{-10}$ & $3.3531 \times 10^{-10}$ \\
\hline
\end{tabular}

of the semiconductor, its failure rate and reliability were computed using (12) and (13), respectively. The reliability, failure rate, and MTTF of the MMC were computed using (15), (16), and (19) respectively. The results are given in Table 4.

\subsection{Case II: results and analysis with CCC}

The rainflow analysis is repeated when the CCC is invoked, and the corresponding rainflow histogram of the temperature swings is shown in Fig. 5. Results for the life consumption of the semiconductor switch under this condition are given in Table 5. With the CCC, the total life consumption due to temperature swings is $7.1026 \times 10^{-10} \%$, which yields an expected lifetime of 446.2271 years. The failure rate and reliability index of the converter when it is operated with the CCC are computed in the same way. The results are shown
Table 6 Lifetime and reliability of a converter with CCC

\begin{tabular}{llr}
\hline Parameter & Designation & \multicolumn{1}{c}{ Value } \\
\hline MTTF for IGBT & $\mu_{\text {IGBT }}$ (years) & 446.2271 \\
IGBT failure rate & $\lambda_{\text {IGBT }}$ & 0.0022 \\
IGBT reliability & $R_{\mathrm{IGBT}}$ & 0.9978 \\
MMC reliability & $R_{\mathrm{MMC}}$ & 0.9569 \\
MMC failure rate & $\lambda_{\mathrm{MMC}}$ & 0.0441 \\
MTTF for MMC & $\mu_{\mathrm{MMC}}$ (years) & 22.6757 \\
\hline
\end{tabular}

Table 7 Experimental setup parameters

\begin{tabular}{ll}
\hline Parameter & Value \\
\hline Number of submodule per arm & 4 \\
Submodule capacitance & $4.7 \mathrm{mF}$ \\
Arm inductance & $1 \mathrm{mH}$ \\
Load resistor & $5 \Omega$ \\
Load inductor & $4 \mathrm{mH}$ \\
DC link voltage & $100 \mathrm{~V}$ \\
Carrier frequency & $4 \mathrm{kHz}$ \\
Modulation index & 0.9 \\
\hline
\end{tabular}

in Table 6. Table 5 and Table 6 show that the thermal minimization capability of the CCC results in a $3.06 \%$ increase in the reliability index and a $68.25 \%$ increase in the expected lifetime of the MMC.

\section{Experimental results}

For the purpose of validating the simulation results, a scaled down laboratory prototype MMC was developed based on the system parameters shown in Table 7. The structure of the experimental setup is shown in Fig. 6. The MMC prototype is a single-phase converter with four SMs per arm. A discrete IGBT (GT20J341) [40] is used in building the prototype. The MMC control is implemented using a Texas Instrument TMS320F28377D Dual-Core Delfino ${ }^{\mathrm{TM}}$ microcontroller board. The coding of the control strategy is implemented with code composer studio.

\subsection{Case I: experimental results without CCC}

The electrical behavior of a converter without the proposed CCC is shown in Fig. 7. The presence of double-line frequency component can be clearly seen on the arm currents, as shown in Fig. 7a, and on circulating current, as shown in Fig. 7c, of the converter. The multilevel arm voltages are unaffected. The output AC current and voltage are also unaffected as shown in Fig. $7 b$. 


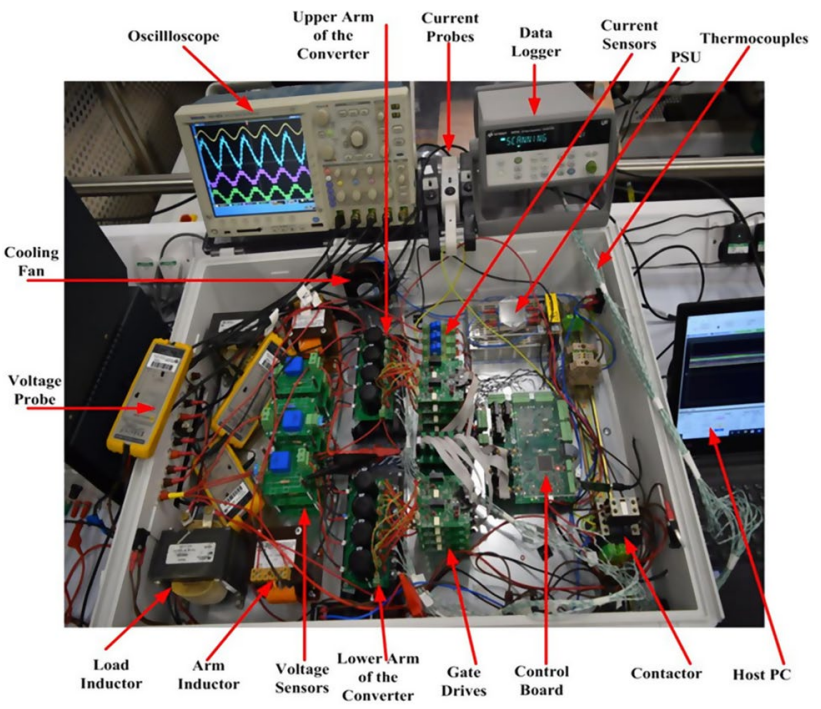

Fig. 6 Experimental setup

(a)

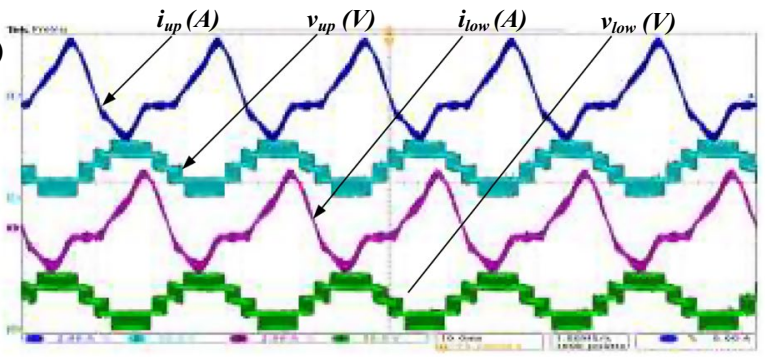

(b)

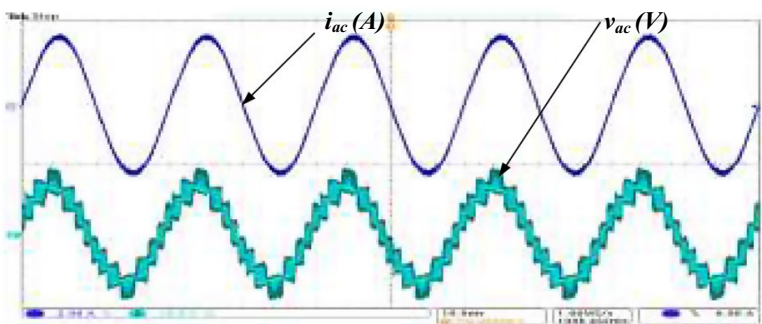

(c)

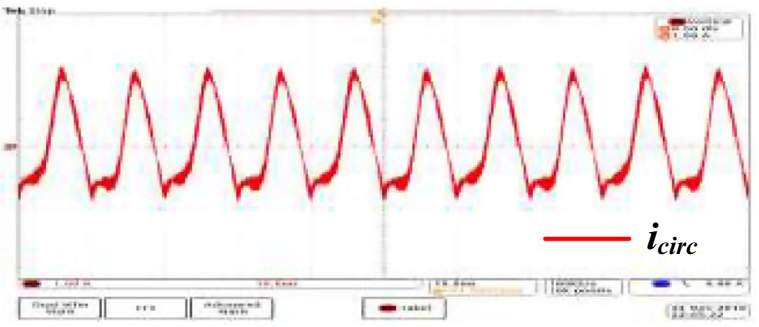

Fig. 7 Electrical behavior of a converter without CCC: a arm currents and voltages; $\mathbf{b}$ output current and voltage; $\mathbf{c}$ circulating current

\subsection{Case II: experimental results with CCC}

Figure 8 shows the electrical behavior of a converter when (a)

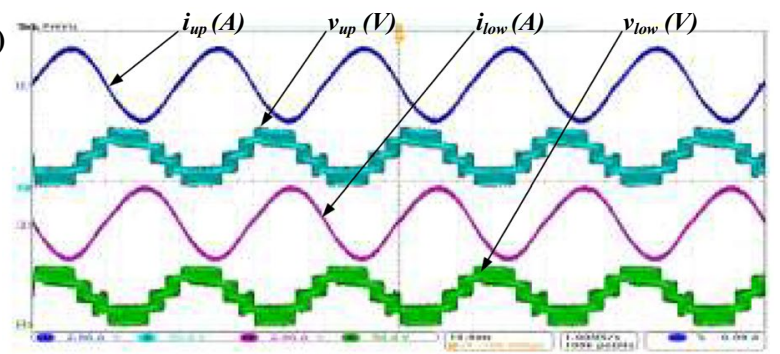

(b)

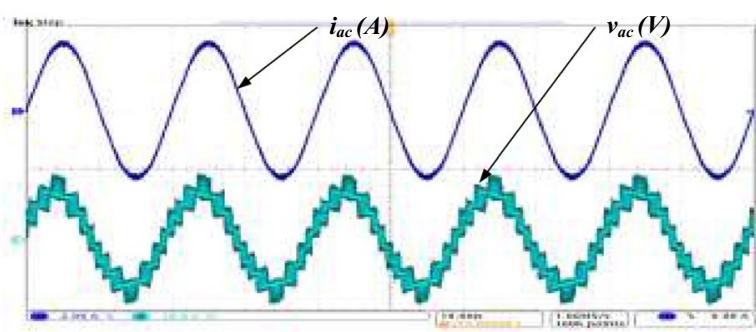

(c)

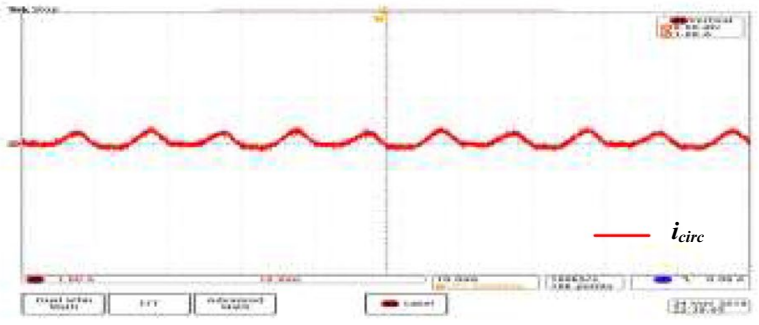

Fig. 8 Electrical behavior of a converter with CCC: a arm currents and voltages; $\mathbf{b}$ output current and voltage; $\mathbf{c}$ circulating current

the proposed CCC is incorporated. As shown in Fig. 8a, the controller action is able to suppress the double-line frequency component on the arm currents. The amplitude of the circulating current, as shown in Fig. 8c, decreased significantly, and the second harmonic component has been significantly suppressed, which results in a converter with higher reliability and lifetime expectancy.

\section{Conclusion}

In this paper, a controlled circulating current injection method has been proposed as a means of improving the lifetime and reliability of an MMC. The proposed method is based on adding the controlled differential voltage from the closed loop CCC as an offset to the reference modulating signals of the converter arms. A model for estimating the junction temperature profile of the semiconductor devices has been developed and a rainflow algorithm was applied to the turning points of the temperature. An analytical model was developed based on the output of the rainflow algorithm in conjunction with the Coffin-Manson-Arrhenius law and Miner's rule for calculation of the lifetime and reliability indices of the converter with and without the proposed 
control strategy. Simulation and experimental results demonstrate the effectiveness of the proposed method. As such, the proposed method can be used as a control freedom for enhancing the lifetime and reliability of MMCs.

Open Access This article is licensed under a Creative Commons Attribution 4.0 International License, which permits use, sharing, adaptation, distribution and reproduction in any medium or format, as long as you give appropriate credit to the original author(s) and the source, provide a link to the Creative Commons licence, and indicate if changes were made. The images or other third party material in this article are included in the article's Creative Commons licence, unless indicated otherwise in a credit line to the material. If material is not included in the article's Creative Commons licence and your intended use is not permitted by statutory regulation or exceeds the permitted use, you will need to obtain permission directly from the copyright holder. To view a copy of this licence, visit http://creativecommons.org/licenses/by/4.0/.

\section{References}

1. Lesnicar, A., Marquardt, R.: A new modular voltage source inverter topology. In: Proc. of Eur. Conf. on Power Electron. Appl. Toulouse, France (2003)

2. Han, X., Sima, W., Yang, M., Li, L., Yuan, T., Si, Y.: Transient characteristics under fround and short-circuit faults in a $\pm 500 \mathrm{kV}$ MMC_based HVDC system with hybrid DC circuit breakrs. IEEE Trans. Power Del. 33(3), 1378-1887 (2018)

3. Hao, Q., Li, Z., Gao, F., Zhang, J.: Reduced-order small-signal models of modular multilevel converter and MMC-based HVdc grid. IEEE Trans. Ind. Electron. 66(3), 2257-2268 (2019)

4. Zhu, R., Lin, N., Dinavashi, V., Liang, G.: An accurate and fast method for conducted EMI modeling and simulation of MMCbased HVdc converter station. IEEE Trans. Power Electron. 35(5), 4689-4702 (2020)

5. Isik, S., Anurag, A., Bhattacharya, S.: Modeling of MMC based FACTS device as a replacement of UPFC for power flow and oscillation damping control. 46th Annual Conf. of IEEE Ind. Electrons. Society, pp. 4188-4193 (2020)

6. Li, H. et al: Hardware design of a $1.7 \mathrm{kV} \mathrm{SiC} \mathrm{MOSFET} \mathrm{based}$ MMC for medium voltage motor drives. Appl. Power Electrons. Conf. Expo, pp. 1649-1655 (2018)

7. Li, Y., et al.: PLL synchronization stability analysis of MMCconnected wind farms under high-impedance AC faults. IEEE Trans. Power Syst. 36(3), 2251-2261 (2021)

8. Khanal, S., Disfani, V.: 'Modular multilevel converter design for grid integration of solar photovoltaic systems. IEEE power and energy society general meeting, pp. 1-5 (2020)

9. Kadandani, N.B., Dahidah, M., Ethni, S.: Design and development of modular multilevel converter for solid state transformer application. Bayero J. Eng. Technol. 16(1), 31-41 (2021)

10. Pérez-Basante, A., et al.: Circulating current control for modular multilevel converters with $(\mathrm{N}+1)$ selective harmonic elimination-PWM. IEEE Trans. Power Electron. 35(8), 8712-8725 (2020)

11. Chen, X., Liu, J., Song, S., Ouyang, S.: Circulating harmonic currents suppression of level-increased NLM based modular multilevel converter with deadbeat control. IEEE Trans. Power Electron. 35(11), 11418-11429 (2020)

12. Tanta, M., et al.: Deadbeat predictive current control for circulating currents reduction in a modular multilevel converter based rail power conditioner. Appl. Sci. 10, 1-22 (2020)
13. Zhang, H., Wickramasinghe, H.R., Jing, L., Li, J., Pou, J., Konstantinou, G.: Circulating current control scheme of modular multilevel converters supplying passive networks under unbalanced load conditions. Electr. Power Syst. Res. 171, 36-46 (2019)

14. Bakhshizadeh, M.K., Ma, K., Loh, P.C., Blaabjerg, F.: Indirect thermal control for improved reliability of modular multilevel converter by utilizing circulating current. IEEE applied power electron. conf. and exp. Charlotte, NC, 2167-2173 (2015)

15. Tu, Q., Xu, Z., Xu, L.: Reduced switching-frequency modulation and circulating current suppression for modular multilevel converters. IEEE Trans. Power Del. 6(3), 2009-2017 (2011)

16. Hagiwara, M., Akagi, H.: PWM control and experiment of modular multilevel converters. Power Electron. Spec. Conf, pp. 154161 (2008)

17. Gonçalves, J., Rogers, D.J., Liang, J.: Submodule temperature regulation and balancing in modular multilevel converters. IEEE Trans. Ind. Electron. 65(9), 7085-7094 (2018)

18. Hahn, F., Andresen, M., Buticchi, G., Liserre, M.: Thermal analysis and balancing for modular multilevel converters in HVDC applications. IEEE Trans. Power Electron. 33(3), 1985-1996 (2018)

19. Andresen, M., Ma, K., Carne, G.D., Buticchi, G., Blaabjerg, F., Liserre, M.: Thermal stress analysis of medium-voltage converters for smart transformers. IEEE Trans. Power Electron. 32(6), 4753-4765 (2017)

20. Held, M., Jacob, P., Nicoletti, G., Scacco, P., Poech, M.: Fast power cycling test of IGBT modules in traction application. 2nd int. conf. on power electron. and drive syst, Singapore, 1, 425-430 (1997)

21. Nielsen, R. Ø., Due, J., Munk-Nielsen, S.: Innovative measuring system for wear-out indication of high power IGBT modules. IEEE energy conv. cong. and expo, pp. 1785-1790 (2011)

22. Huang, H., Mawby, P.A.: A lifetime estimation technique for voltage source inverters. IEEE Trans. Power Electron. 28(8), 41134119 (2013)

23. GopiReddy, L.R., Tolbert, L.M., Ozpineci, B., Pinto, J.O.P.: Rainflow algorithm-based lifetime estimation of power semiconductors in utility applications. IEEE Trans. Ind. Appl. 51(4), 3368-3375 (2015)

24. Bannantine, J.A., Comer, J.J., Handrock, J.L.: Fundamentals of Metal Fatigue Analysis. Prentice-Hall, Englewood Cliffs (1990)

25. Perpina, X., Jorda, X., Vellvehi, M., Rebollo, J., Mermet-Guyennet, M.: Long-term reliability of railway power inverters cooled by heat-pipe based systems. IEEE Trans. Ind. Electron. 58(7), 2662-2672 (2011)

26. Miner, M.A.: Cumulative damage in fatigue. J. Appl. Mech. 12, A159-A164 (1945)

27. Wang, H., et al.: Transitioning to physics-of-failure as a reliability driver in power electronics. IEEE J. Emerg. Sel. Top. Power Electron. 2(1), 97-114 (2014)

28. Hirschmann, D., Tissen, D., Schröder, S., Doncker, R.W.D.: Reliability prediction for inverters in hybrid electrical vehicles. IEEE Trans. Power Electron. 22(6), 2511-2517 (2007)

29. Bryant, A.T., Mawby, P.A., Palmer, P.R., Santi, E., Hudgins, J.L.: Exploration of device reliability using compact device models and fast electrothermal simulation. IEEE Trans. Ind. Appl. 44(3), 894-903 (2008)

30. Yang, S., Bryant, A., Mawby, P., Xiang, D., Ran, L., Tavner, P.: An Industry-based survey of reliability in power electronic converters. IEEE Trans. Ind. Appl. 47(3), 1441-1451 (2011)

31. Choi, U., Blaabjerg, F., Lee, K.: Study and handling methods of power IGBT module failures in power electronic converter systems. IEEE Trans. Power Electron. 30(5), 2517-2533 (2015)

32. Infineon: IGBT Module FF75R12RT4 Data Sheet (2013) 
33. Musallam, M., Johnson, C.M.: An efficient implementation of the rainflow counting algorithm for life consumption estimation. IEEE Trans. Reliab. 61(4), 978-986 (2012)

34. Jones, D.R.H., Ashby, M.F.: Engineering materials 1-an introduction to properties, applications and design, 3rd edn, Oxford (2015)

35. Stephens, P.I., Fatemi, A., Stephens, R.R., Fuchs, H.O.: Metal fatigue in engineering, 2nd edn. Wiley, Hoboken (2000)

36. Nieslony, A.: Determination of fragments of multiaxial service loading strongly influencing the fatigue of machine components. Mech. Syst. Signal Process. 23, 712-2721 (2009)

37. Graditi G., Adinolfi, G.: Energy performances and reliability evaluation of an optimized DMPPT boost converter. Int. Conf. on Clean Electr. Power, Ischia, Italy, 69-72 (2011)

38. Richardeau, F., Pham, T.T.L.: Reliability calculation of multilevel converters: theory and applications. IEEE Trans. Ind. Electron. 60(10), 4225-4233 (2013)

39. Xu, X., Zhao, P., Zhao, C.: Reliability analysis and redundancy configuration of MMC with hybrid submodule topologies. IEEE Trans. Power Electron. 31(4), 2720-2729 (2016)

40. Toshiba: IGBT GT20J341 Datashet (2012)

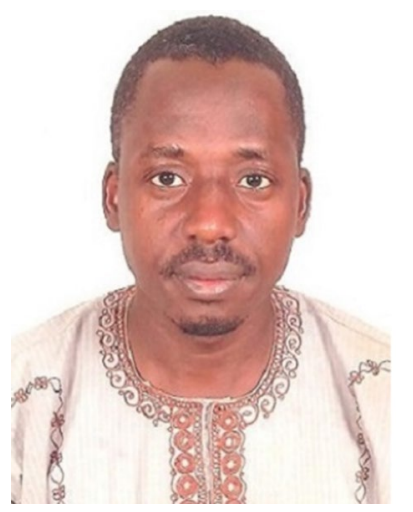

Nasiru B. Kadandani received his B.Eng. and M.Eng. degrees in Electrical Engineering from Bayero University Kano, Kano, Nigeria, in 2004 and 2010, respectively. He received his $\mathrm{PhD}$ degree in Electrical Engineering from Newcastle University, Newcastle upon Tyne, England, UK, in 2021. Dr. Kadandani is presently with the Electrical Power Research Group, School of Engineering, Newcastle University. He is a registered Member of the Council for the Regulation of Engineering in Nigeria (COREN); and the Nigerian Society of Engineers (NSE). Dr. Kadandani was the Nigerian representative during the 9th International Training on Wind Turbine Technology and Applications held at the Centre for Wind Energy Technology (C-WET), Chennai, India, in 2012. He has authored or coauthored over 20 articles published in referenced journals and conference proceedings. His current research interests include multilevel converters, solid-state transformers, and grid integration of renewable energy.

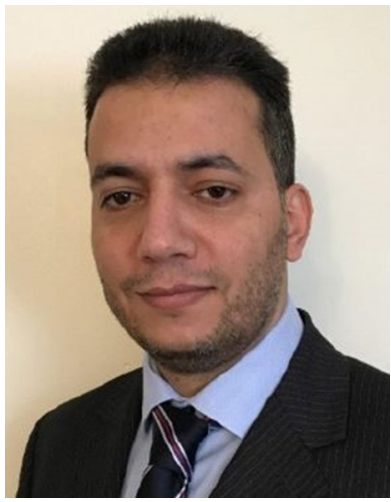

Mohamed Dahidah received his $\mathrm{PhD}$ degree in Electrical Engineering from the Multimedia University, Malacca, Malaysia, in 2008. Dr Dahidah was an Assistant Professor in the Department of Electrical and Electronic Engineering, University of Nottingham, Malaysia Campus, until November 2012. $\mathrm{He}$ is presently working as a Senior Lecturer in the School of Engineering, Newcastle University, Newcastle upon
Tyne, England, UK. Dr Dahidah was a recipient of a first prize paper award at the IEEE Conference on Sustainable Utilization and Development in Engineering and Technology, Kuala Lumpur, Malaysia, in 2010; and a Girling Watson Fellowship Award from the University of Sydney, Sydney, Australia, in 2009. He has authored or co-authored over 80 referenced papers published in journals and conference proceedings in the field of power electronics. His current research interests include modular power converters, battery chargers for EVs, solid-state transformers, and advanced power conversion for renewable energy integration. Dr. Dahidah is the Deputy Editor-in-Chief for IET Power Electronics and has been a regular reviewer for both IEEE and IET journals.

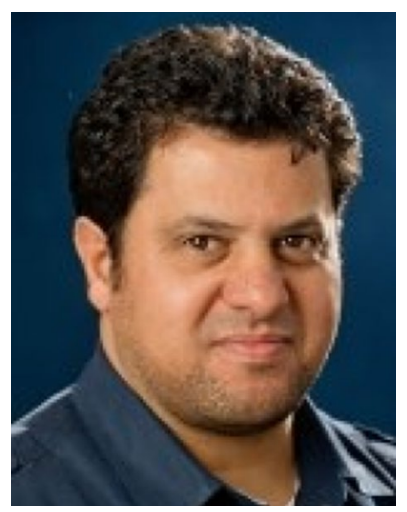

Salaheddine Ethni received his B.Sc. (Hons) degree in Electrical Engineering from the Bright Star University of Technology, Brega, Libya, in 1995, specializing in Automatic Control. He received his M.Sc. degree in Automation and Control, and his $\mathrm{PhD}$ degree in Electrical Engineering from Newcastle University, Newcastle upon Tyne, England, UK, in 2004 and 2011, respectively. He joined the Electrical Power Research Group, School of Engineering, Newcastle University, in September 2013. He is presently working as a Lecturer in the School of Engineering, Newcastle University. He has authored or co-authored a number of referenced articles published in journals and conference proceedings. His current research interests include nature-inspired optimization algorithms, modeling and control of electric vehicle batteries, control of electric drives, system identification and parameter estimation of power electronic systems, electric power generation, condition monitoring and fault diagnosis of electrical machines, power electronic converters, adaptive control of power electronic systems, and stand-alone and grid-connected PV systems.

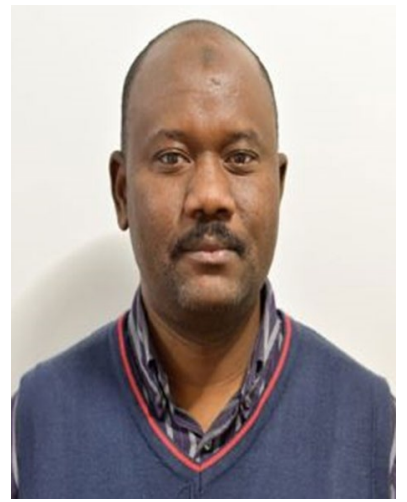

Musbahu Muhammad received his B.Eng. and M.Eng. degrees in Electrical and Electronics Engineering from Bayero University Kano, Kano, Nigeria, in 2001 and 2010, respectively. $\mathrm{He}$ received his $\mathrm{PhD}$ degree Electrical and Electronics Engineering from Newcastle University, Newcastle upon Tyne, England, $\mathrm{UK}$, in 2017 . He is presently working as a Lecturer of electronics and control at Teesside University Middlesbrough, England, UK. He has more than 9 years of experience in the fields of high-gain dc-dc power converters, soft-switching techniques, and power converter modeling and control. His current research interests include battery management systems (BMS) and Li-ion battery characterization, prognosis, and diagnosis. 